\title{
The Effect of Orlistat as an Adjuvant to Lifestyle Modification on Weight Loss in Polycystic Ovary Syndrome Patients:An Evidence-Based Case Report
}

\author{
Nobian Andre ${ }^{1}$, Kevin Aristyo ${ }^{1}$, Aqila Sakina Zhafira ${ }^{1}$, Riesta Hanjani ${ }^{1}$, \\ Diannisa Paramita Susantono ${ }^{1}$, Rachel Ethelind ${ }^{1}$, Listya Tresnanti Mirtha ${ }^{2}$ \\ ${ }^{I}$ Faculty of Medicine Universitas Indonesia, Jakarta, Indonesia \\ ${ }^{2}$ Department of Community Medicine, Faculty of Medicine Universitas Indonesia, Jakarta, Indonesia
}

\begin{abstract}
Polycystic ovary syndrome (PCOS) occurs in 1 of 10 women of reproductive age. This syndrome is highly associated with obesity. Therefore, many of the patients face weight loss challenges. Given that many patients find it rather difficult to change their lifestyle, medicamentous intervention poses an option. Orlistat is an inhibitor of carboxyl ester lipase which inhibits the hydrolysis of dietary triglycerides that decreases the absorption of fatty acids and monoglyceride. The purpose of this study is to determine whether administration of orlistat can improve diet and exercise-induced body weight reduction in PCOS patients with obesity. Literature searching was done in 3 databases: PubMed, Cochrane Library, and Embase which yielded 141 articles. Findings were narrowed down using duplicate removal, inclusion and exclusion criteria into two relevant articles of randomized controlled trials. One randomized clinical trial showed a greater percentage in weight loss in PCOS patients BMI $>23 \mathrm{~kg} / \mathrm{m} 2$ administered with orlistat and lifestyle modification in comparison to lifestyle modification alone $(7.81 \%(6.51-9.11 \%)$ vs $4.7 \%(4.19-5.21 \%)$; $\mathrm{p}<0.001)$. Similar body weight reduction was also found by another randomized clinical trial in PCOS patients BMI $>25 \mathrm{~kg} / \mathrm{m} 2$ with orlistat treatment (from $81.5 \mathrm{~kg}(80.3-82.7)$ to $76.2 \mathrm{~kg}(74.92-77.48)$ vs from $80.91 \mathrm{~kg}(79.65-82.17)$ to $79.15 \mathrm{~kg}(77.8-80.5) ; \mathrm{p}<0.01)$. It was concluded that a considerable reduction of weight in obese and overweight PCOS patients was found when given orlistat with exercise and dietary interventions compared to exercise and dietary interventions alone.
\end{abstract}

Keywords - orlistat, polycystic ovary syndrome, weight loss

\section{INTRODUCTION}

Polycystic ovary syndrome (PCOS) is a common endocrine disorder in women of reproductive age. It is estimated to affect 1 in 10 women of childbearing age.[1] This disorder is characterized by the symptoms of dysmenorrhea, infertility, hirsutism, and is strongly associated with weight gain, especially central obesity [1,2].

Obesity is a prevalent feature in PCOS as it is observable in 42-74\% of PCOS patients [3-5]. Obesity is clinically defined as an increased body mass index (BMI) that ranges between $30-34.9 \mathrm{~kg} / \mathrm{m}^{2}$ [6]. This condition is a multifactorial event mainly as a result of a positive energy balance, arising from excessive nutrient intake, lack of physical activity and with influence from genetic factors [7]. Relating back to PCOS, whether this disorder is the main causative factor of obesity is yet to be established. However, studies have suggested the mechanism relating PCOS and obesity that is through a decreased thermogenesis in postprandial state and the dysregulation of ghrelin signals in PCOS patients [8,9].Unfortunately, there is no cure for PCOS and the management needed to prevent health problems is given based on symptoms (palliative) [1]. To this day, standard treatment for obesity in PCOS is adapted from type II diabetic patients, which is through lifestyle modifications [10,11]. The management involves hypocaloric diet, a specialized type of weight loss-diet, that reduces calorie intake from $200 \mathrm{kcal} /$ day to $1000 \mathrm{kcal} /$ day and is effective to reduce body weight by $7-10 \%$. To accompany that, it is also recommended for obese, PCOS patients to exercise minimally 30 minutes per day for 3-5 times/week [10,11]. Given that many patients find it rather difficult to change their lifestyle, medicamentous intervention poses an option. Orlistat is an US Food and Drug Administration (FDA)-approved drug that acts on (particularly inhibits) carboxyl ester lipase to prevent the hydrolysis of triglycerides into free fatty acid and monoglyceride $[12,13]$. Thus, it decreases the absorption of dietary fat. It is currently used as an anti-obesity drug that is combined with low-fat diet as well as regular exercise. Several side effects have been identified with the consumption of orlistat, which include increased bowel movement, oily stool, oily spotting, and stomach pain [12]. Several studies have shown the effectiveness of orlistat in weight reduction in obese patients [12,13]. This EBCR intends to understand whether orlistat administration is appropriate for weight reduction in obese, PCOS patients. 


\subsection{Case Illustration}

A 35-year-old woman came to a clinic complaining about failure to lose weight. Her body weight is $75 \mathrm{~kg}$, height $155 \mathrm{~cm}$ (body mass index $=31$ ). She also felt stressed from her irregular menstruation cycle and excessive facial hair. She was previously diagnosed with Polycystic Ovary Syndrome (PCOS) and PCOSrelated weight gain. To manage her obesity, she was told to regularly exercise and control her diet in order to lose weight. She has been doing those things for a while but she felt like her body weight did not improve much. That is due to the fact that she works as a corporate worker for 8 hours a day, 5 days a week, which often hampers her exercise schedule. She usually spends most of the day sitting in her office. However, she did not have any problem with diet control. Recently, she heard about a drug to treat obesity, called orlistat. The drug was advertised to result in weight loss. Since the drug is not cheap for her, she came to the clinic and asked the doctor whether orlistat can really aid her effort to lose weight.

\subsection{Clinical Question Formulation}

Based on the information from the presented case, the formulation of clinical question and PICO framework are as follows:

In PCOS patients with obesity, would the addition of orlistat to controlled diet and exercise improve weight loss in comparison to controlled diet and exercise alone?

- Patients $(\mathrm{P}) \quad$ : PCOS patients with obesity

- Intervention (I) : Orlistat with exercise and diet control

- Comparison (C): Placebo with exercise and diet control

- Outcome (O) : Weight loss

We aim to determine whether administration of orlistat can improve diet and exercise-induced body weight reduction in PCOS patients with obesity.

\section{METHODS}

\subsection{Search strategy}

Literature searching was performed in three databases: PubMed, Cochrane Library, and Embase on $22^{\text {nd }}$ September 2016. The terms found in PICO — along with their synonyms and related terms-were translated into MeSH terms and text words to be used as keywords in each database, which are listed in Table 1. Subsequently, search yield was filtered through a selection process displayed by our flowchart (Figure 1). The inclusion and exclusion criteria are also shown in the flowchart.

Table 1. Search strategy used in Cochrane Library, PubMed, and Embase (conducted on September $22^{\text {nd }} 2016$ )

\begin{tabular}{|l|l|c|c|}
\hline Database & \multicolumn{1}{|c|}{ Search Terms } & Hits & $\begin{array}{c}\text { Selected } \\
\text { article(s) }\end{array}$ \\
\hline $\begin{array}{l}\text { Cochrane } \\
\text { Library }\end{array}$ & $\begin{array}{l}\text { (orlistat [Title/Abstract/Keywords] OR xenical } \\
\text { [Title/Abstract//eywords] OR alli } \\
\text { [Title/Abstract/Keywords] OR tetrahydrolipstatin } \\
\text { [Title/Abstract/Keywords]) AND (weight } \\
\text { loss[Title/Abstract/Keywords/MeSH Terms] OR body } \\
\text { size[Title/Abstract/Keywords/MeSH Terms]) AND } \\
\text { (Polycystic Ovary } \\
\text { Syndrome[Title/Abstract/Keywords/MeSH Terms]) }\end{array}$ & 9 & 0 \\
\hline PubMed & $\begin{array}{l}\text { (orlistat[Title/Abstract] OR xenical[Title/Abstract] OR } \\
\text { alli[Title/Abstract] OR } \\
\text { tetrahydrolipstatin[Title/Abstract]) AND (weight } \\
\text { loss[Title/Abstract/MeSH Terms] OR body } \\
\text { size[Title/Abstract/MeSH Terms]) AND (Polycystic } \\
\text { Ovary Syndrome[Title/Abstract/MeSH Terms]) }\end{array}$ & 17 & 1 \\
\hline Embase & $\begin{array}{l}\text { (orlistat [Title/Abstract] OR xenical [Title/Abstract] } \\
\text { OR alli [Title/Abstract] OR tetrahydrolipstatin } \\
\text { [Title/Abstract]) AND (weight loss [Title/Abstract] } \\
\text { OR waist circumference [Title/Abstract] OR weight } \\
\text { reduction [Title/Abstract] OR body size } \\
\text { [Title/Abstract]) AND (Polycystic Ovary } \\
\text { Syndrome[Title/Abstract/MeSH Terms]) }\end{array}$ & 115 & 1 \\
\hline
\end{tabular}




\subsection{Critical Appraisal}

Following literature selection, the relevant articles were appraised by consensus of all authors using critical appraisal tool for therapeutic study from Centre for Evidence-Based Medicine (CEBM), Oxford University. The checklists were obtained from http://www.cebm.net/critical-appraisal/ and covers validity, importance, and applicability aspects. The critical appraisal results are shown in Table 2.

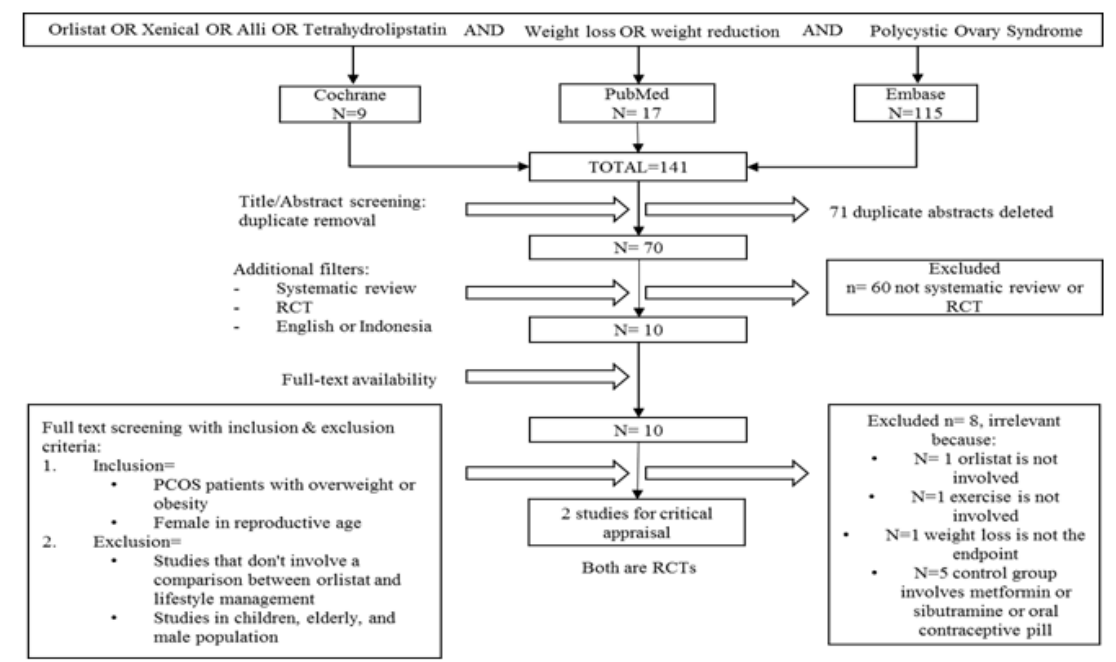

Figure 1. Selection flowchart. Articles were filtered by duplicate removal, study design, language, and selection criteria. We opted for randomized controlled trial (RCT) or systematic review as both studies have a high level of evidence for therapy studies. Inclusion and exclusion criteria are listed in the above figure.

\subsection{Critical Appraisal}

\section{RESULTS}

This evidence-based case report will review the effectiveness of orlistat as an add-on to controlled diet and exercise in reducing body weight in PCOS patients with obesity compared to controlled diet and exercise alone. The outcomes of interest for this review include reduction in body weight, body mass index (BMI), waist circumference (WC), and waist-hip ratio (WHR). From the search selection and filtration above, two eligible RCT articles were selected to be reviewed: Kumar P, Arora S 2014 \& Moini et al. 2015. Both articles reported the use of orlistat for body weight reduction in PCOS-related obesity.

Table 2. Critical appraisal of the two studies based on criteria by CEBM Oxford University

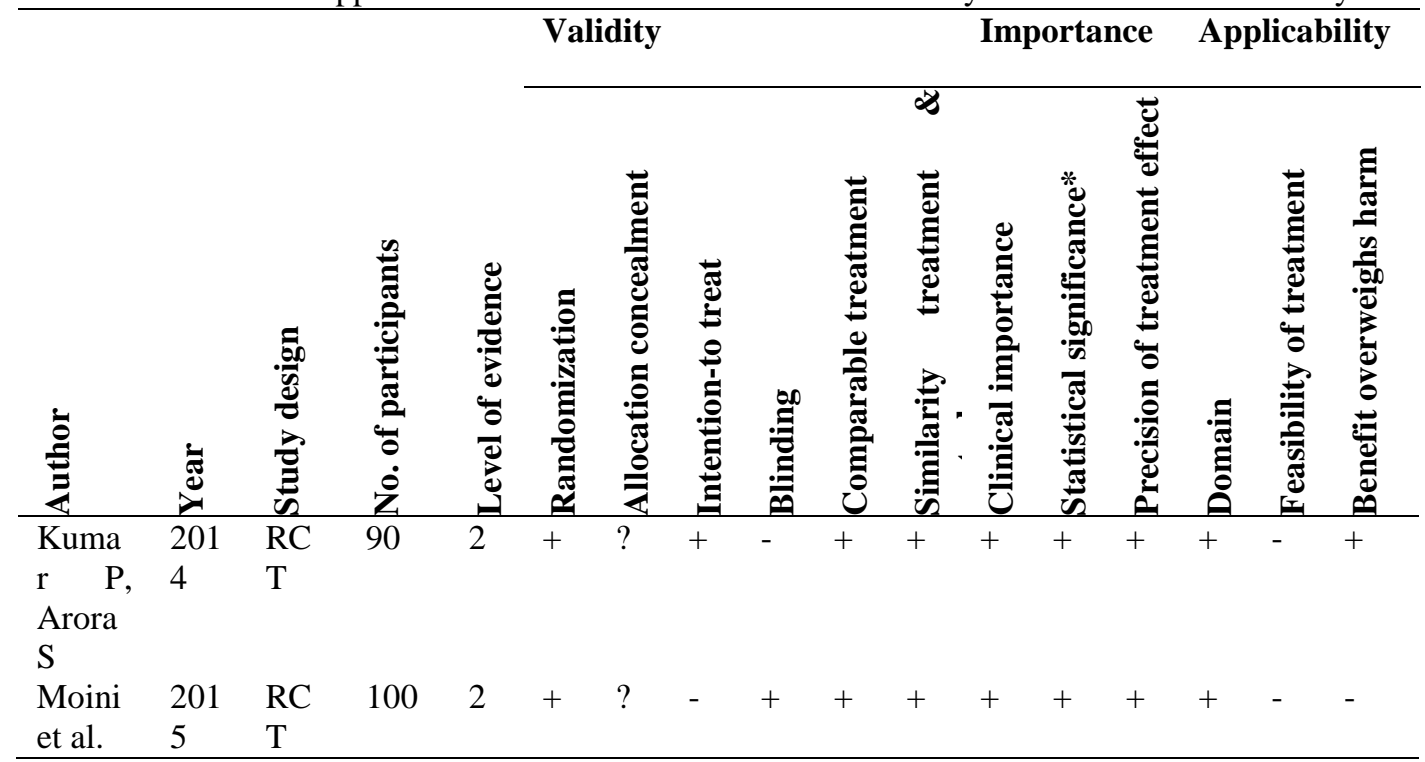

+ mentioned clearly; - not done; ? not clear; *statistical significance at $\mathrm{p}<0.05$ 
Both selected studies were critically appraised for their validity, importance, and applicability. Both studies from Kumar and Arora [14] and Moini et al [15] failed to mention allocation method. Nevertheless, the two studies fulfilled 4 out of 6 validity criteria, thus regarded as valid. Furthermore, both studies are considered important as they presented statistically significant difference $(\mathrm{p}<0.05)$ between treatment and control groups, with non-overlapping, narrow confidence interval. This information is displayed in Table 3. In terms of applicability, we found that the valid and important studies may not be highly applicable in our setting. The reason being is that although the age group and clinical characteristics were similar to that of our setting (that is, women of reproductive age with PCOS-related obesity), we found several issues regarding different ethnicity which potentially causes different dietary habit, the side effects reported by Moini et al [15], and drug's feasibility. Orlistat is readily available in Indonesia yet it is rather expensive. Certainly, this aspect of applicability is highly subjective and needs to be discussed further with the patient.

\subsection{The Evidence}

The study done by Kumar and Arora [14] was a randomized-controlled trial in India that included 90 female aged below 40 years old with PCOS and had BMI $\geq 23 \mathrm{~kg} / \mathrm{m}^{2}$. PCOS was determined using 2003 Rotterdam criteria. Participants were randomized into orlistat $(n=30)$, metformin $(n=30)$ or control $(n=30)$ groups using block randomization. Note that we only assessed the comparison between the orlistat group and the control group. All participants were given hypocaloric diet consisting of 1,200-1,800 kcal/day, accompanied by 1-hour exercise every day. The exercise was organized by a physiotherapist. In addition to hypocaloric diet and exercise, orlistat group received $120 \mathrm{mg}$ orlistat twice a day, whereas the control group only received hypocaloric diet and exercise alone. Treatment duration was three months The outcomes assessed in this study include reduction in weight, BMI, WC, WHR, ovulation rates, conception rates, and lipid profile. There were statistically significant differences in reductions in weight, BMI, WC, and WHR between orlistat and control groups (Table 3). There were no adverse events reported. This study was conducted in India, which might entail different dietary habit compared to Indonesian setting.

Moini et al. [15] conducted a randomized, double-blind, controlled trial, which involved 100 patients that were randomized into two groups using randomization table method. Participants recruited were female aging between 19-38 years old with PCOS and BMI $>25 \mathrm{~kg} / \mathrm{m}^{2}$. PCOS was determined using 2004 Rotterdam criteria. All participants received hypocaloric diet consists of 1,200-1,800 kcal/day and were encouraged to walk for 30 minutes each day. Intervention group $(n=50)$ was given orlistat $(120 \mathrm{mg})$ three times per day, whereas control group $(n=50)$ was given placebo. Treatment duration was three months, followed-up monthly with a blinded assessor. Outcomes assessed in this study were weight loss, BMI, WHR, testosterone, fasting insulin, fasting blood glucose, HOMA-IR, and lipid profile. There were statistically significant reduction in weight, BMI, and WHR between intervention and control groups (Table 3). Moini et al. [15] also reported about adverse events experienced by $50 \%$ of the patients in the intervention group including oily spotting, oily stools, and headache. In contrast to previous study, this study might be more similar to Indonesia in terms of dietary habit as it was conducted in Iran.

Table 3. The summary of results of the two studies

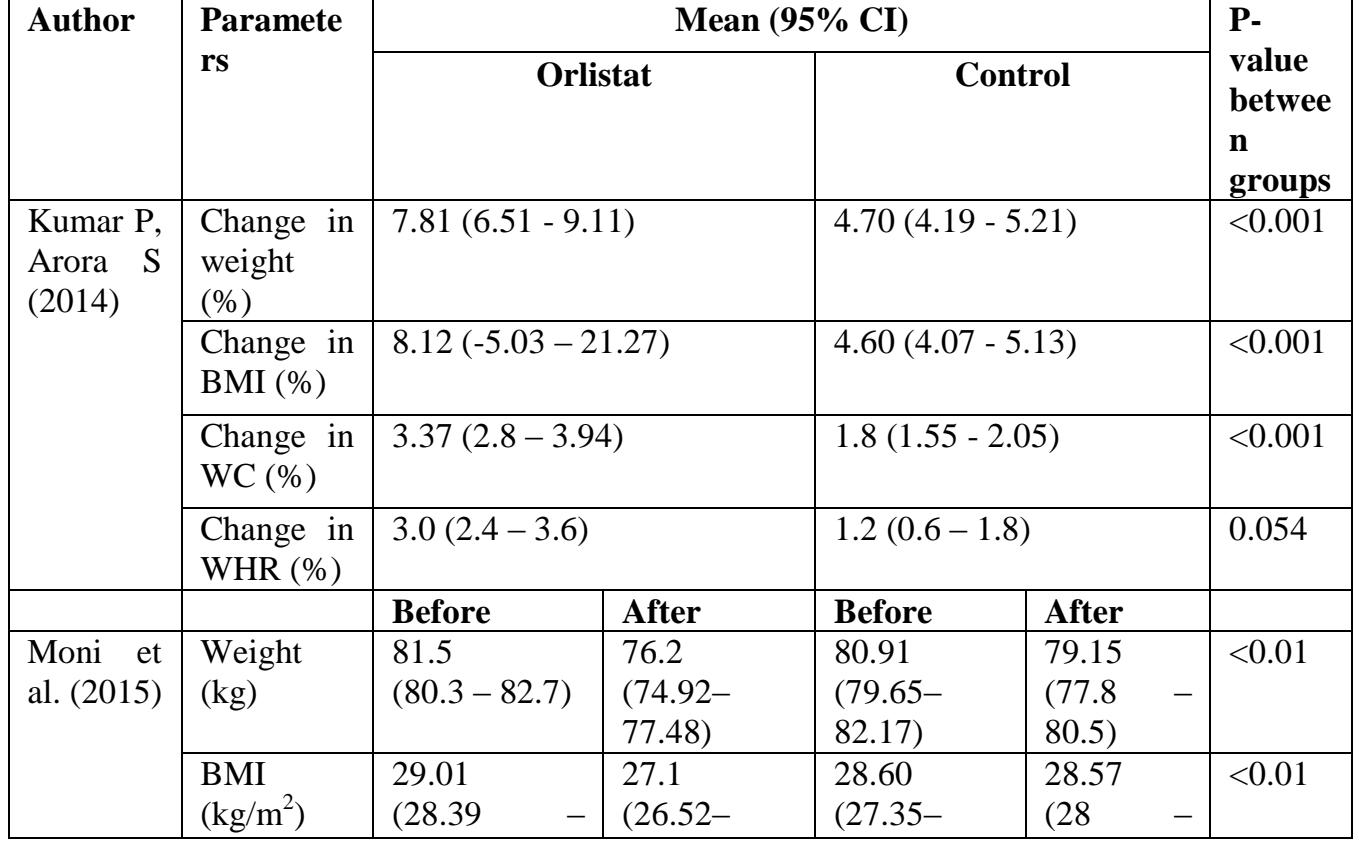




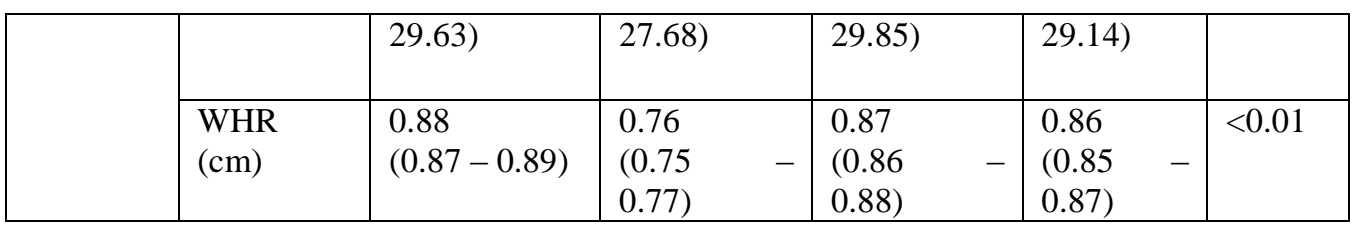

$\mathrm{BMI}=$ body mass index $\mathrm{WC}=$ waist circumference; $\mathrm{WHR}=$ waist-hip ratio; $\mathrm{P}$-values were obtained from ANOVA statistical test.

\section{DISCUSSION}

As mentioned previously, there is currently no cure for PCOS. This also applies to the weight loss challenge faced by a majority of PCOS patients. Generally, diet and lifestyle management are the conventional first-lines of weight loss strategy in obese patients. Additional interventions using orlistat in obese patients suffering from PCOS are currently being put into practice. In this report, the relevant articles obtained were only two studies. We believe that this is due to a large heterogeneity among studies in the topic of PCOS-related obesity (i.e.: some involved metformin, contraceptive pills, some did not include orlistat, etc.). That being said, we still managed to find high quality evidence that is appropriate for our case.The two studies have several strong and weak points in terms of study design. The study by Kumar and Arora [14] was considered as a strong study given that it was an RCT. Furthermore, the recruited patients were based on an established tool, properly randomized to minimize confounding factors, well maintained that they were completely followed up (allowing intention-to-treat analysis). However, they used small sample size and did not use blinding method, making the study susceptible to observer bias. The study by Moini et al. [15] was also considered strong, had a proper recruitment process and randomization for the same reasons as above. In addition, they also utilized double blinding method to prevent observer bias. However, they had poor participant maintenance shown by some loss to follow up and withdrawals.We found that the results from the two RCTs are in line with one another. Although the first study presented their results in percent changes (\%) and the second study presented their results in before-after measurements, they both agree on statistically significant reduction in body weight $(\mathrm{p}<0.01)$ and BMI $(\mathrm{p}<0.01)$ in PCOS patients consuming orlistat compared to controls. Moreover, both studies are comparable as they used similar number of patients (Kumar used $n=30$ in each group while Moini ended up with $n=43$ in each group), similar tool for patient recruitment (Rotterdam criteria), and both used 3 monthtreatment period $[14,15]$. However, the two studies found different significance for the WHR although both agree that greater reduction occurred in orlistat group.Interestingly, from clinical point of view, the amount of weight loss in study by Kumar and Arora was more pronounced than in Moini et al.'s ( $8 \%$ and $6 \%$ respectively) despite of the more frequent $120 \mathrm{mg}$ orlistat administration in the latter study (twice a day and three times a day respectively). This might be attributed to the difference in physical activity program engaged by the study participants. Kumar and Arora prescribed a mandatory aerobic exercise for 1 hour each day [14] while Moini and colleagues only encouraged the patients to walk for 30 minutes daily (with only $70 \%$ compliance rate) [15]. In addition, if we look more to the study setting, Kumar and Arora [14] conducted the trial in India in which the majority of diet consist of vegetables. This type of diet might aid the attempt to reduce weight. In contrast, Moini et al. [15] conducted the trial in Iran where most diets are of meat and poultry (higher fat content). This type of diet together with the more frequent dosage that they used might also be responsible for the adverse events experienced by $50 \%$ of the intervention group.

Based on the evidence and our analysis, it is apparent that orlistat can work in conjunction with controlled-diet and exercise to reduce body weight and BMI. However, it may not take over and have the main role. Frequent administration of orlistat with infrequent or even no exercise is not likely to result in favorable outcome and may also lead to the occurrence of side effects (e.g.: steatorrhea). It is essential to educate the patient that in order for orlistat to give an optimal result, she needs to exercise regularly and control her diet. She is encouraged to consume orlistat until her BMI is decreased to $28 \mathrm{~kg} / \mathrm{m}^{2}$ as orlistat is only allowed for patients with BMI over $28 \mathrm{~kg} / \mathrm{m}^{2}$ [16]. More importantly, it is pertinent to discuss with the patient about the orlistat cost, patient's values and preferences to assess benefit-risk ratio. Also, physicians should inform the patient to avoid orlistat during pregnancy if she is planning to have one because orlistat is assigned to pregnancy category $\mathrm{X}$ by the FDA.According to the stages of change, lifestyle modification should be incorporated into the "action phase" [17]. If the patient is successful in practicing this new behavior for at least six months, she will be able to enter "maintenance phase" in which she is able to incorporate this lifestyle into her life to prevent relapse [18]. In our case, this means that in order for the patient to maintain her BMI even after stopping orlistat consumption, she needs to practice regular exercise and controlled-diet for at least six months. After the BMI is down to $28 \mathrm{~kg} / \mathrm{m}^{2}$, orlistat should be withdrawn and we expect the patient to continue her new lifestyle leading to body weight conservation. 


\section{v. CONCLUSION}

In conclusion, studies exhibit a considerable reduction of weight in obese and overweight PCOS patients when given orlistat with exercise and dietary interventions compared to exercise and dietary interventions alone. Both studies were performed with valid methodology and presented important results. However the applicability to our patients is arguable due to the differences of race and ethnicity, which does not only imply a difference in genetics but also in dietary intake tendencies as a result of cultural influence.

Translating this report into clinical practice, we recommend administration of orlistat to obese and overweight PCOS patients (BMI $>28 \mathrm{~kg} / \mathrm{m} 2$ ) as an adjunct to their exercise and dietary intervention, but not as a stand-alone treatment. It is important to adjust the frequency of orlistat administration with the degree of exercise and dietary intervention, as orlistat may give rise to unfavorable side effects.

\section{REFERENCES}

[1] Office on Women's Health. Polycystic ovary syndrome [internet]. [updated: June 8 2016; cited: Oct 2 2016].Available from: http://www.womenshealth.gov/publications/our-publications/fact-sheet/polycysticovary-syndrome.html

[2] Pal L. Polycystic ovary syndrome: current and emerging concepts. New York: Springer; 2014.

[3] Nestler JE. Metformin for the treatment of the polycystic ovary syndrome. N Engl J Med. 2008;358:4754.

[4] Azziz R, Woods KS, Reyna R, Key TJ, Knochenhauer ES, Yildiz BO. The prevalence and features of the polycystic ovary syndrome in an unselected population. Clin Endocrinol Metab. 2004;89:2745-9.

[5] Azziz R, Sanchez LA, Knochenhauer ES, Moran C, Lazenby J, Stephens KC, et al. Androgen excess in women: experience with over 1000 consecutive patients. Clin Endocrinol Metab. 2004;89:453-62.

[6] Bray GA. Pathophysiology of obesity. Am J Clin Nutr. 1992;5(2 Suppl):488S-94.

[7] Swinburn BA, Sacks G, Hall KD, McPherson K, Finegood DT, Moodie ML, et al. The global obesity pandemic: shaped by global drivers and local environments. Lancet. 2011;378(9793): 804-14.

[8] Segal KR, Dunaif A. Resting metabolic rate and postprandial thermogenesis in polycystic ovarian syndrome. Int J Obes. 1990;14:559-67.

[9] Moran LJ, Noakes M, Clifton PM, Wittert GA, Le Roux CW, Ghatei MA, et al. Postprandial ghrelin, cholecystokinin, peptide YY, and appetite before and after weight loss in overweight women with and without polycystic ovary syndrome. Am J Clin Nutr. 2007;86:1603-10.

[10] Lieoa GU, Sengupta A, Karsies D. Polycystic ovary syndrome (PCOS) and other androgen excess-lated conditions; can changes in dietary intake make a difference? Nutr Clin Pract. 2008 Feb;23(1):63-71

[11] Moran LJ, Pasquali R, Teede HJ, Hoeger KM, Norman RJ. Treatment of obesity in polycystic ovary syndrome : a position statement of the Androgen Excess and Polycystic Ovary Syndrome Society. Fertil Steril. 2009 Dec;92(6):1966-82

[12] Orlistat: MedlinePlus Drug Information [Internet]. Medlineplus.gov. 2016 [cited: Sep 15 2016]. Available from: https://medlineplus.gov/druginfo/meds/a601244.html

[13] Saunders K, Shukla A, Igel L, Kumar R, Aronne L. Pharmacotherapy for Obesity. Endocrinology and Metabolism Clinics of North America. 2016;45(3):521-538.

[14] Kumar P, Arora S. Orlistat in polycystic ovarian syndrome reduces weight with improvement in lipid profile and pregnancy rates. Journal of Human Reproductive Sciences. 2014;7(4):255-62

[15] Moini A, Kanani M, Kashani L, Hoseini R, and Hosseini L. Effect of orlistat on weight loss, hormonal, and metabolic profiles in women with polycystic ovarian syndrome: a randomized double-blind placebocontrolled trial. Endocrine. 2015;49:286-9.

[16] Practice Guidance: OTC Orlistat [Internet]. Royal Pharmaceutical Society of Great Britain. 2000 [cited: Sep 28 2016]. Available from: https://www.rpharms.com/support-pdfs/rps-practice-guidance-otcorlistat.pdf

[17] Bosworth G. A 'Stages of Change' Approach to Helping Patients Change Behavior - American Family Physician [Internet]. Aafp.org. 2000 [cited: Sep 28 2016]. Available at: http://www.aafp.org/afp/2000/0301/p1409.html

[18] Harvard Medical School. Why it's hard to change unhealthy behavior - and why you should keep trying [internet]. [updated: unknown; cited: Oct 2 2016]. Available from: http://www.health.harvard.edu/staying-healthy/why-its-hard-to-change-unhealthy-behavior 